\title{
The Burden of Laboratory-confirmed Influenza Infection in Lebanon Between 2008 and 2016: A single tertiary care center experience
}

\section{Aia Assaf-Casals}

American University of Beirut Medical Center

\section{Zeina Saleh}

American University of Beirut Medical Center

\section{Sarah Khafaja}

American University of Beirut Medical Center

Danielle Fayad

American University of Beirut Medical Center

\section{Hady Ezzeddine}

American University of Beirut Medical Center

\section{Mohammad Saleh}

American University of Beirut Medical Center

\section{Sarah Chamseddine}

American University of Beirut Medical Center

\section{Rouba Sayegh}

American University of Beirut Medical Center

\section{Sima Sharara}

American University of Beirut Medical Center

\section{Ahmad Chmaisse}

American University of Beirut Medical Center

\section{Souha Kanj}

American University of Beirut Medical Center

\section{Zeina Kanafani}

American University of Beirut Medical Center

Rima Hanna-Wakim

American University of Beirut Medical Center

\section{George Araj}

American University of Beirut Medical Center

\section{Rami Mahfouz}

American University of Beirut Medical Center

\section{Reiko Saito}

Niigata Daigaku 
Hiroshi Suzuki

Niigata Daigaku

Hassan Zaraket

American University of Beirut Medical Center

Ghassan Dbaibo ( $\sim$ gdbaibo@aub.edu.lb )

https://orcid.org/0000-0002-5813-5878

\section{Research article}

Keywords: Influenza, Hospitalization, Morbidity, Mortality, Antiviral use

Posted Date: February 4th, 2020

DOI: https://doi.org/10.21203/rs.2.22568/v1

License: (우 (i) This work is licensed under a Creative Commons Attribution 4.0 International License. Read Full License

Version of Record: A version of this preprint was published at BMC Infectious Diseases on May 12th, 2020.

See the published version at https://doi.org/10.1186/s12879-020-05013-7. 


\section{Abstract}

BACKGROUND: Influenza is a major cause of morbidity and mortality worldwide. Following the 2009 pandemic, there was widened interest in studying influenza burden in all regions. However, data from the World Health Organization (WHO) Middle East and North Africa (MENA) region remain limited particularly from Lebanon.

METHODS: A retrospective chart review extending over a period of 8 seasons from Jan 1st, 2008 till June 30 th , 2016 at a tertiary care center in Beirut was performed. All laboratory-confirmed cases of influenza were included for analysis. Data on epidemiology, clinical presentation, complications, antiviral use and mortality were collected for analysis.

RESULTS: A total number of 1829 cases of laboratory-confirmed influenza were identified. Average annual positivity rate was $14 \%$. Both influenza $A$ and $B$ co-circulated in each season with predominance of influenza A. Influenza virus started circulating in December and peaked in January and February. The age group of 1950 years accounted for the largest proportion of cases (22.5\%) followed by the age group of 5-19 years (18\%). Pneumonia was the most common complication reported in $33 \%$ of cases. Mortality reached $3.8 \%$. The two extremes of age ( $<2$ years and $\geq 65$ years) were associated with a more severe course of disease, hospitalization, intensive care unit (ICU) admission, complications, and mortality rate. Of all the identified cases, $26 \%$ were hospitalized. Moderate-to-severe disease was more likely in influenza B cases but no difference in mortality was reported between the two types. Antivirals were prescribed in $68.8 \%$ and antibiotics in $41 \%$ of cases. There seemed to be an increasing trend in the number of diagnosed and hospitalized cases over the years of the study.

CONCLUSION: Influenza causes a substantial hospitalization burden annually in Lebanon with significant morbidity across all age groups. A population based prospective surveillance study is needed to better estimate the burden of Influenza in Lebanon that would help formulate a policy on influenza control.

\section{Introduction}

Acute respiratory infections constitute a major cause of morbidity and mortality globally.(1) According to the updated 2004 World Health Organization (WHO) report, the death toll from acute lower respiratory infections caused by bacterial and viral infections reached 4.2 million and accounted for $7.2 \%$ of all deaths worldwide. $(1,2)$ Data from the Global Burden of Disease study estimated that the mortality rate from lower respiratory tract infections due to influenza reached 0.9 per 100,000 population in the WHO Middle East and North Africa (MENA) region and the hospitalization rate ranged from 100 to 299 per 100,000 population.(3)

Influenza is a major cause of both influenza-like illness (ILI) and more importantly severe acute respiratory infection (SARI) across the globe. $(4,5)$ Several studies in different regions of the world have indicated that influenza is a significant underlying cause for hospitalization of patients with acute respiratory infection (ARI).(6-9) 
Infection with influenza may manifest in a wide spectrum of illness levels ranging from asymptomatic infection to death. Different studies reported asymptomatic cases to account for $14 \%$ up to $77 \%$ of influenza infections.(10-12)The major cause for hospital admission due to influenza is the development of pneumonia, which could be either primary viral or secondary to bacterial co-infection, and acute respiratory distress syndrome. $(13,14)$ In severe cases of influenza infection, death may ensue even in the absence of comorbidities.(15)

Annual seasonal influenza vaccination can reduce the chances of getting infected and subsequently prevent spread of the virus. Vaccines are available in two forms: the inactivated influenza vaccine (IIV), which is administered intramuscularly or intradermally, and the live attenuated influenza vaccine, administered intranasally, (LAIV). Vaccination is recommended by the Centers for Disease Control, USA, annually to everyone above 6 months of age and should be given before influenza season commences, usually during early fall or late summer.(16)However, in many countries, especially developing ones, there is a lack of data on the epidemiology of influenza disease leading to a lack of appreciation of its true burden by public health authorities and impeding the introduction of influenza vaccine on national vaccination schedules.

Accordingly, the WHO recommended the generation of country-specific epidemiological data in order to identify high priority groups, emphasizing the inclusion of pregnant women, and the optimal timing of vaccination against influenza. (17)

Given the epidemic nature of the illness, there has been an increasing interest in the burden resulting from its outbreaks in addition to concerns about its association with myocardial infarctions, glycemic variation in diabetic patients, and the exacerbation of other chronic illnesses. $(1,18-27)$ While data on the epidemiology and severity of this disease has been primarily obtained from studies in temperate developed countries, developing regions including the MENA region have also begun investigating this burden in their hospitals allowing the formation of more complete global data.(28)

In Lebanon, the influenza burden of disease is unknown. Since 2008, surveillance of influenza virus among ILI patients at the American University of Beirut Medical Center (AUBMC), was performed at the Center for Infectious Diseases Research (CIDR).(29-33) The Ministry of Public Health started reporting influenza activity as part of its SARI surveillance since 2015 . Whereas these surveillance programs furnish important information about influenza activity in Lebanon, they do not reflect the total burden of disease of influenza. In fact, an ideal study to look at the burden of influenza diseases would be population-based, prospective, and armed with a highly sensitive and specific test for influenza to be conducted both in outpatient and inpatient settings. However, such a study is not feasible and too expensive to conduct. An alternative approach that we chose was to conduct a retrospective review on laboratory-proven influenza at our center where both inpatient and outpatient services exist. The information collected from this review will be helpful in forming a better estimate about the burden of disease of influenza in Lebanon as extrapolated from our findings. This may help inform public policy about prevention of influenza infections.

\section{Methods And Materials}

\section{Study design}


This is a retrospective review of subjects who tested positive for influenza virus at AUBMC, a tertiary care medical center located in Beirut, Lebanon, between January 1, 2008 and June 30, 2016. Samples were collected from these subjects and tested for influenza $A$ and $B$ viruses by rapid diagnostic test and/or by real-time reverse-transcriptase polymerase chain reaction (RT-PCR). Information already collected for subjects diagnosed to have influenza A or B covering all the seasons from 2008 till 2016 who were previously enrolled in three ongoing surveillance studies at the CIDR at AUBMC were also included.

Sampling:

- Samples were either taken by nasopharyngeal swab or nasal wash and tested directly (at bed side - as part of the ILI surveillance studies) using one of the rapid diagnostics test kits or were sent in sterile normal saline to AUBMC laboratory and tested within 24 hours. Deep tracheal aspirates were mostly used for testing patients who were intubated.

- Storage: For those samples obtained from subjects participating in the IRB-approved surveillance studies since 2008, a second sample was taken (after obtaining consent) and was transported in virus transport media, stored at $4 \mathrm{oC}$ for no more than 24 hours or at $-20 \mathrm{oC}$ if taken over the weekend. These samples were ultimately stored at -80 oC until their transfer to the Department of Public Health at Niigata University, Japan for multiplex polymerase chain reaction and viral culture.

Examination of samples:

- Different rapid antigen detection kits were used across the different seasons. These included: Rapid FLU virus test kit Capilia' ${ }^{\text {TM }}$ Tauns, Japan; Quick-Ex flu A/B rapid influenza test Denka Seiken, Japan; Quick-Ex flu A/B \& RSV rapid influenza test Denka Seiken, Japan; and BD Directigen ${ }^{\mathrm{TM}}$ EZ Flu A+B (Becton Dickinson, Cockeysville, Md.). Testing was performed following the manufacturer's instructions.

- Polymerase chain reaction: real-time reverse-transcriptase (RT) polymerase chain reaction (PCR) using the GENEXPERT (Cepheid) platform was used at AUBMC Clinical Molecular Biology Laboratory.(33)

\section{Identification of subjects:}

All subjects were identified retrospectively through medical records at AUBMC and through the parallel ILI surveillance studies for influenza conducted during the study period. These surveillance studies enrolled subjects with symptoms fitting the ILI case definition, presenting as outpatients or inpatients, and tested them for influenza using rapid diagnosis test (RDT) and then RT-PCR. The list of remaining subjects was retrieved by the medical coder by looking at the following lab codes: " 8108 " for rapid influenza A \& B antigen detection and "8988" for Flu PCR. This was done through the Department of Pathology and Laboratory Medicine by sending a Statistical Information Request. Data from each chart was collected and recorded on a case report form (CRF). Medical records with the discharge diagnosis of influenza were also retrieved.

The CRF included information on demographic data (gender, age, residence, etc.), social history, medical history, influenza vaccination history, clinical symptoms, hospital course, severity parameters $\left(\mathrm{O}_{2}\right.$ requirement, intensive care admission, mechanical ventilation, complications), radiology findings, targeted 
laboratory results, samples dates, method of influenza detection, use of antibiotics/antivirals, hospital stay and outcomes.

\section{Inclusion criteria:}

- Any patient regardless of age or hospital admission status who was tested for influenza using either nasal wash/swab followed by PCR or RDT, or by serology at AUBMC lab or who was enrolled in the surveillance studies conducted at CIDR during the time period extending from January 1, 2008 till June 30, 2016 was included in the initial subject count. To note that surveillance studies were interrupted during the 2012/2013 and 2015/2016 seasons. Each influenza season included cases starting July till the end of June of the following year.

- Subjects who tested positive for influenza A, B or both were included for further detailed review and analysis.

\section{Exclusion criteria:}

- Lack of testing for influenza

\section{Definition:}

Moderate to severe influenza disease was defined as (1) fever $>39$ degree Celsius $\left(39^{\circ} \mathrm{C}\right)$, and/or (2) physician-verified shortness of breath, pulmonary congestion, pneumonia, bronchiolitis, bronchitis, wheezing, croup, or acute otitis media, and/or (3) physician-diagnosed serious extra-pulmonary complication of influenza, including myositis, encephalitis, seizure, and/or myocarditis (34).

\section{Statistical analysis:}

The Statistical Package for Social Sciences (SPSS) program, version 22.0 for Windows was used for data analysis (IBM, Armonk, NY). Simple descriptive statistics was used to describe subjects' demographics, morbidity and mortality. Bivariate and multivariate analyses of risk factors for symptomatic influenza infection was analyzed by Pearson's Chi-square test or Fisher's exact test (when the number of subjects in a subgroup was less than 5 ). Continuous risk factors were analyzed with student $t$ test. Statistical significance was considered below a type- 1 error threshold (alpha level) of 0.05 . Following that a multivariate logistic regression model comprised of all risk factors with unadjusted $p$-value $<0.2$ was constructed and reported.

\section{Results}

During the study period spanning 8 influenza seasons from January $1^{\text {st }}, 2008$ till June $30^{\text {th }}, 2016$, a total of 11288 subjects presented to AUBMC with a respiratory illness for which they were tested for influenza infection. Out of all subjects tested, 1829 subjects (16\%) had a positive test result for influenza; 29.6\% (542 
influenza cases) were identified during the 2009 influenza pandemic. Of the 1829 positive cases, 1529 (84\%) were caused by influenza A, 242 cases $(13 \%)$ by influenza B, and 55 cases (3\%) had co-infection with both types (type was not reported in three cases). During the 2009 pandemic, $97.6 \%$ of the infections were caused by influenza $A$ and $2 \%$ were co-infected. However, subtyping was done for only 35 cases during the pandemic, of which 28 cases were caused by influenza A (H1N1)pdm09.

\section{Demographic and clinical characteristics of the subjects}

Of the 1829 cases, 916 (50.1\%) cases were female and 72 cases were pregnant; $24 \%$ of female subjects in the childbearing age (Table 1). Most of the subjects resided in Beirut and Mount Lebanon governorates $(73 \%)$ reflecting the population served by our center. Subjects were divided into 7 age groups: the highest proportion (22.5\%) was for adults between 19 and 50 years old, while only $9.5 \%$ were below 2 years old. At least one underlying comorbidity was present in $45.8 \%$ of cases reviewed and $31 \%$ had two or more comorbidities. The most common underlying chronic disease among adults was cardiovascular diseases (33\%), malignancy (15\%) and diabetes mellitus (14.8\%). However, in the younger age groups (< 19 years) the most common underlying chronic diseases were asthma (16\%) and malignancy (10.4\%). Data on the immunization status against influenza was available for 456 cases; $22.8 \%$ (104) of these subjects had received influenza vaccine in the past 12 months at the time of diagnosis with influenza infection.

The most common presenting symptoms were respiratory (96\%) with cough constituting $91.5 \%$ of these symptoms. Fever was present in $89 \%$ of cases; $78 \%$ of febrile subjects with data on highest temperature recorded had high-grade fever $\geq 39^{\circ} \mathrm{C}$. Gastrointestinal symptoms were present in $33.7 \%$ including vomiting $(47 \%)$, diarrhea (32\%) and abdominal pain (29.6\%). Myalgia was reported in $31 \%$ of subjects. Other less common symptoms (13.6\%) included conjunctivitis, otitis, neurological events, confusion and dizziness.

Table 1. Demographic and clinical characteristics of laboratory-confirmed influenza subjects 


\begin{tabular}{|c|c|}
\hline & Number (\%) \\
\hline \multicolumn{2}{|l|}{ Gender $(n=1829)$} \\
\hline Male & 913 (49.9) \\
\hline Female & $916(50.1)$ \\
\hline \multicolumn{2}{|l|}{ Age groups $(n=1829)$} \\
\hline$[0-2$ years [ & $173(9.5)$ \\
\hline$[2-5$ years [ & $240(13.1)$ \\
\hline [5-10 years [ & $338(18.5)$ \\
\hline$[10-19$ years [ & $328(17.9)$ \\
\hline [19- 50 years [ & $411(22.5)$ \\
\hline$[50-65$ years [ & $164(9.0)$ \\
\hline$\geq 65$ years & $175(9.6)$ \\
\hline \multicolumn{2}{|l|}{ Residence $(n=1829)$} \\
\hline Beirut & $919(50.2)$ \\
\hline Mount Lebanon & $417(22.8)$ \\
\hline South & $79(4.3)$ \\
\hline North & $69(3.8)$ \\
\hline Beqaa & $20(1.1)$ \\
\hline Outside Lebanon & $24(1.3)$ \\
\hline Unknown & $301(16.5)$ \\
\hline Presence of underlying comorbidities ( $n=1303$ ) & $597(45.8)$ \\
\hline \multicolumn{2}{|l|}{ Number of underlying comorbidities $(n=1303$ ) } \\
\hline None & $706(54.2)$ \\
\hline One & $412(31.6)$ \\
\hline More or equal to 2 & $185(14.2)$ \\
\hline \multicolumn{2}{|l|}{ Underlying comorbidities ( $n=1303$ ) } \\
\hline Cardiovascular disease & $222(17.0)$ \\
\hline Chronic pulmonary disease & $54(4.1)$ \\
\hline Asthma & $160(12.3)$ \\
\hline Immunosuppressive state (other than malignancy) & $19(1.5)$ \\
\hline
\end{tabular}




\begin{tabular}{|c|c|}
\hline Diabetes Mellitus & $96(7.4)$ \\
\hline Renal disease & $52(4.0)$ \\
\hline Neuromuscular disorders & $12(0.9)$ \\
\hline Neurological disorders & $58(4.5)$ \\
\hline Malignancy & $164(12.6)$ \\
\hline Hemoglobinopathy & $30(2.3)$ \\
\hline \multicolumn{2}{|c|}{ Days from onset to swabbing $n=1368$ ) } \\
\hline 0-2 days & $882(64.5)$ \\
\hline 3-4 days & $338(24.7)$ \\
\hline$>4$ days & $148(10.8)$ \\
\hline Influenza Vaccine $(n=456)$ & $104(22.8)$ \\
\hline
\end{tabular}

Complications secondary to influenza infection were captured when available. Almost $24 \%(295 / 1245)$ of subjects developed at least one complication. Pneumonia, confirmed by the presence of infiltrates or consolidation on radiological examination, was the most common complication $(81.6 \% ; 241 / 295)$. Chest Xray was done in 233 of these cases and detected the presence of pneumonia in 218 cases $(90.4 \%)$; the remaining 15 subjects $(6.6 \%)$ had a clear chest X-ray but had abnormal findings on computed tomography (CT). In eight subjects, only a CT was done initially and detected the presence of pneumonia. However, the treating physician did not request an imaging study in 1095 subjects $(60 \%)$. When imaging was requested, pneumonia was detected in $32.8 \%$ of cases $(241 / 734)$. The highest rate of pneumonia cases was in the older age groups: $\geq 65$ years old $(27.4 \%)$ and $50-65$ years $(19.1 \%)(P$-value $=0.000)$. Other reported complications were croup ( 3 cases), shock (20 cases) of which $20 \%$ were below 2 years and $25 \%$ were above 65 years of age, sepsis ( 23 cases) of which $39 \%$ were $\geq 65$ years, myocardial infarction ( 4 cases), renal failure (12 cases, all adults), acute respiratory distress syndrome (16 cases, $25 \%$ were $<2$ years and $37.5 \%$ were between 19 and 50 years old), encephalopathy (6 cases), hemorrhagic pneumonia ( 1 case), stroke (2 cases), seizures (7 cases) and liver failure ( 5 cases; all adults).

Moderate to severe influenza was encountered in 591 cases $(42 \%)$ with a significant P-value $(<0.001)$ between different age groups. Almost half of the subjects below 5 years $(50.5 \%)$ and above 50 years $(51.6 \%)$ had moderate to severe disease while around a third (28\%) of those 10 to 50 years did (Table 2). When comparing percentages of moderate to severe disease within each type of influenza detected, influenza B infection was associated with a slightly higher incidence (49.8\%) of moderate to severe disease compared to influenza A infection $(40.9 \%)(P$-value $=0.054)$.

Table 2. Severity parameters of laboratory-confirmed influenza cases according to different age groups 


\begin{tabular}{|c|c|c|c|c|c|c|c|c|c|}
\hline & $\begin{array}{l}\text { Total } \\
\text { n (\%) }\end{array}$ & $\begin{array}{l}{[0-2[} \\
n(\%)\end{array}$ & $\begin{array}{l}{[2-5[} \\
n(\%)\end{array}$ & $\begin{array}{l}\text { [5-10[ } \\
\mathrm{n}(\%)\end{array}$ & $\begin{array}{l}{[10-} \\
19[ \\
\text { n (\%) }\end{array}$ & $\begin{array}{l}{[19-50[} \\
\text { n (\%) }\end{array}$ & $\begin{array}{l}{[50-} \\
65[ \\
\text { n (\%) }\end{array}$ & $\begin{array}{l}\geq 65 \\
n(\%)\end{array}$ & $\begin{array}{l}\mathrm{p}- \\
\text { value }\end{array}$ \\
\hline $\begin{array}{l}\text { Moderate to } \\
\text { severe disease } \\
(\mathrm{N}=1399)\end{array}$ & $\begin{array}{l}591 \\
(42.2)\end{array}$ & $\begin{array}{l}80 \\
(51.9)\end{array}$ & $\begin{array}{l}103 \\
(52.0)\end{array}$ & $\begin{array}{l}95 \\
(45.5)\end{array}$ & $\begin{array}{l}60 \\
(28.0)\end{array}$ & $\begin{array}{l}91 \\
(29.3)\end{array}$ & $\begin{array}{l}73 \\
(49.7)\end{array}$ & $\begin{array}{l}89 \\
(53.6)\end{array}$ & $<0.001$ \\
\hline $\begin{array}{l}\text { Hospitalization } \\
\text { rate }(\mathrm{N}=1787)\end{array}$ & $\begin{array}{l}475 \\
(26.6)\end{array}$ & $\begin{array}{l}57 \\
(34.5)\end{array}$ & $\begin{array}{l}37 \\
(16.2)\end{array}$ & $\begin{array}{l}24 \\
(7.3)\end{array}$ & $\begin{array}{l}24 \\
(7.5)\end{array}$ & $\begin{array}{l}103 \\
(25.5)\end{array}$ & $\begin{array}{l}99 \\
(60.4)\end{array}$ & $\begin{array}{l}131 \\
(74.9)\end{array}$ & $<0.001$ \\
\hline $\begin{array}{l}\text { ICU admission } \\
(\mathrm{N}=1804)\end{array}$ & $\begin{array}{l}70 \\
(3.9)\end{array}$ & $\begin{array}{l}13 \\
(7.6)\end{array}$ & $\begin{array}{l}6 \\
(2.5)\end{array}$ & $\begin{array}{l}1 \\
(0.3)\end{array}$ & $\begin{array}{l}2 \\
(0.6)\end{array}$ & $\begin{array}{l}14 \\
(3.5)\end{array}$ & $\begin{array}{l}11 \\
(6.7)\end{array}$ & $\begin{array}{l}23 \\
(13.2)\end{array}$ & $<0.001$ \\
\hline $\begin{array}{l}\text { Oxygen } \\
\text { supplementation } \\
(\mathrm{N}=1616)\end{array}$ & $\begin{array}{l}121 \\
(7.5)\end{array}$ & $\begin{array}{l}13 \\
(9.4)\end{array}$ & $\begin{array}{l}8 \\
(4.1)\end{array}$ & $\begin{array}{l}6 \\
(2.1)\end{array}$ & $\begin{array}{l}3 \\
(1.1)\end{array}$ & $\begin{array}{l}17 \\
(4.4)\end{array}$ & $\begin{array}{l}25 \\
(15.5)\end{array}$ & $\begin{array}{l}49 \\
(28.3)\end{array}$ & $<0.001$ \\
\hline $\begin{array}{l}\text { Mechanical } \\
\text { ventilation } \\
(\mathrm{N}=1804)\end{array}$ & $\begin{array}{l}35 \\
(1.9)\end{array}$ & $8(4.7)$ & $\begin{array}{l}5 \\
(2.1)\end{array}$ & $\begin{array}{l}1 \\
(0.3)\end{array}$ & $\begin{array}{l}2 \\
(0.6)\end{array}$ & $8(2.0)$ & $\begin{array}{l}4 \\
(2.4)\end{array}$ & $\begin{array}{l}7 \\
(4.0)\end{array}$ & $0.002^{\dagger}$ \\
\hline $\begin{array}{l}\text { Complication } \\
\text { rate }(\mathrm{N}=1245)\end{array}$ & $\begin{array}{l}295 \\
(23.7)\end{array}$ & $\begin{array}{l}45 \\
(34.4)\end{array}$ & $\begin{array}{l}36 \\
(22.1)\end{array}$ & $\begin{array}{l}20 \\
(11.6)\end{array}$ & $\begin{array}{l}16 \\
(9.6)\end{array}$ & $\begin{array}{l}51 \\
(16.9)\end{array}$ & $\begin{array}{l}52 \\
(35.6)\end{array}$ & $\begin{array}{l}75 \\
(45.5)\end{array}$ & $<0.001$ \\
\hline \multirow{2}{*}{$\begin{array}{l}\text { Mortality rate in } \\
\text { hospitalized } \\
\text { patients ( } N=469)\end{array}$} & $\begin{array}{l}18 \\
(3.8)\end{array}$ & $2(3.6)$ & $\begin{array}{l}2 \\
(5.7)\end{array}$ & $\begin{array}{l}0 \\
(0.0)\end{array}$ & $\begin{array}{l}0 \\
(0.0)\end{array}$ & $3(2.9)$ & $\begin{array}{l}5 \\
(5.1)\end{array}$ & $\begin{array}{l}6 \\
(4.6)\end{array}$ & $0.909^{\dagger}$ \\
\hline & $\begin{array}{l}\text { Total } \\
\text { mean } \\
\text { days } \\
( \pm S D)\end{array}$ & $\begin{array}{l}{[0-2[} \\
\text { mean } \\
\text { days } \\
( \pm S D)\end{array}$ & $\begin{array}{l}{[2-5[} \\
\text { mean } \\
\text { days } \\
( \pm S D)\end{array}$ & $\begin{array}{l}{[5-} \\
10[ \\
\text { mean } \\
\text { days } \\
( \pm S D)\end{array}$ & $\begin{array}{l}{[10-} \\
19[ \\
\text { mean } \\
\text { days } \\
( \pm S D)\end{array}$ & $\begin{array}{l}{[19-50[} \\
\text { mean } \\
\text { days } \\
( \pm S D)\end{array}$ & $\begin{array}{l}{[50-} \\
65[ \\
\text { mean } \\
\text { days } \\
( \pm S D)\end{array}$ & $\begin{array}{l}\geq 65 \\
\text { mean } \\
\text { days } \\
( \pm S D)\end{array}$ & $\begin{array}{l}\mathrm{p}- \\
\text { value }\end{array}$ \\
\hline $\begin{array}{l}\text { Duration of ICU } \\
\text { stay }(\mathrm{N}=70)\end{array}$ & $\begin{array}{l}9.2 \\
( \pm 8.9)\end{array}$ & $\begin{array}{l}8.2 \\
( \pm 5.2)\end{array}$ & $\begin{array}{l}8.7 \\
(8.8)\end{array}$ & $22^{*}$ & $\begin{array}{l}5.5 \\
( \pm 6.4)\end{array}$ & $\begin{array}{l}12.6 \\
( \pm 12.3)\end{array}$ & $\begin{array}{l}7.7 \\
( \pm 6.6)\end{array}$ & $\begin{array}{l}8.2 \\
(9.3)\end{array}$ & 0.533 \\
\hline $\begin{array}{l}\text { Duration of } \\
\text { hospital stay } \\
(\mathrm{N}=473)^{\star \star}\end{array}$ & $\begin{array}{l}7.0 \\
( \pm 8.8)\end{array}$ & $\begin{array}{l}8.5 \\
( \pm 10.3)\end{array}$ & $\begin{array}{l}8.1 \\
( \pm 8.7)\end{array}$ & $\begin{array}{l}7.2 \\
(13.1)\end{array}$ & $\begin{array}{l}6.6 \\
( \pm 5.0)\end{array}$ & $\begin{array}{l}6.5 \\
( \pm 8.7)\end{array}$ & $\begin{array}{l}6.4 \\
( \pm 6.8)\end{array}$ & $\begin{array}{l}7.0 \\
( \pm 8.8)\end{array}$ & 0.788 \\
\hline
\end{tabular}

Pearson's Chi-Square test was used (no expected count less than 5). Fisher's exact test was used when expected count was less than 5

*Only one patient was admitted to ICU in this age group

** Two patients were excluded; one stayed in 113 days and the other stayed for 262 days related to their underlying medical condition (Neuromuscular disease and cerebral palsy)

To note that a different denominator was used to calculate the rate of each variable. The denominator depended on the number of cases with available data on the studied variable. 


\section{Progression of illness and in-hospital outcome of laboratory- confirmed influenza subjects}

A total of 475 cases $(26.6 \%, N=1787)$ required hospital admission for management. Table (2) shows a comparison of the course of hospital stay among the different age groups with laboratory-confirmed influenza. The highest hospitalization rates were among the elderly $\geq 65$ years $(74.3 \%)$, older adults aged $50-65$ years $(60.4 \%)$, followed by children $<2$ years old (34.5\%). The mean duration of hospital stay was 7 days and there was no statistically significant difference between the different age groups (P-value $=0.788)$. In total, 70 (3.9\%) subjects required ICU admission for management of influenza-related complications. The highest rates of these admissions were at both age extremes; $13.2 \%$ of the elderly $\geq 65$ years and $7.6 \%$ of children $<2$ years with a significant $P$-value $<0.001$ between the different age groups. However, there was no significant difference in the mean duration of ICU stay for all age groups ( $P$-value $=0.533$ ). Oxygen supplementation was required for 121 subjects $(7.5 \%)$ of which 35 cases ultimately required mechanical ventilation. There was a significant statistical difference among different age groups concerning oxygen supplementation and mechanical ventilation with the highest percentages for oxygen supplementation requirement among the elderly $\geq 65$ years $(28.3 \%)$ while the highest percentages for mechanical ventilation was among children $<2$ years $(4.7 \%)$.

Since follow up was not documented for outpatients, outcome was only reported in 469 hospitalized patients, out of which 18 cases (3.8\%) died during their hospital stay. Influenza type A was the detected type in 15 out of the 18 mortality cases, while Influenza type B was detected in the remaining 3 cases. Eleven cases $(69 \%)$ were older than 50 years and three cases were young adults. Only 4 cases were in the pediatric age group ( $<5$ years old) with influenza A detected in all four cases. All cases with fatal outcome had at least one comorbid condition except for one case who was one month old. This case was also co-infected with respiratory syncytial virus. Another 7 cases also had laboratory proven viral co-infections (Cytomegalovirus (CMV), Epstein Barr Virus (EBV) and Herpes Simplex Virus Type-1 (HSV-1); five of whom had an underlying malignancy, one had an underlying neurological disease and one was a healthy pregnant female.

Table 3. Risk factors for mortality in cases of laboratory proven influenza in hospitalized subjects 


\begin{tabular}{|c|c|c|c|}
\hline Characteristic & $\begin{array}{l}\text { Recovery, } \\
\text { n (\%) N=451 }\end{array}$ & $\begin{array}{l}\text { Death, } \\
\text { n (\%) N=18 }\end{array}$ & p-value \\
\hline Age group & & & $0.909 *$ \\
\hline [0-2 years [ & $54(12.0)$ & $2(11.1)$ & \\
\hline [2- 5 years [ & $33(7.3)$ & $2(11.1)$ & \\
\hline [5-10 years [ & $24(5.3)$ & $0(0.0)$ & \\
\hline$[10-19$ years [ & $24(5.3)$ & $0(0.0)$ & \\
\hline [19- 50 years [ & $99(22.0)$ & $3(16.7)$ & \\
\hline$[50-65$ years [ & $93(20.6)$ & $5(27.8)$ & \\
\hline$\geq 65$ years & $124(27.5)$ & $6(33.3)$ & \\
\hline Gender & & & 0.611 \\
\hline Male & $228(50.6)$ & $8(44.4)$ & \\
\hline Female & $223(49.4)$ & $10(55.6)$ & \\
\hline Presence of underlying conditions & $318(70.5)$ & $16(88.9)$ & 0.091 \\
\hline \multicolumn{4}{|l|}{ Underlying medical conditions } \\
\hline Cardiovascular disease & $161(35.7)$ & $8(44.4)$ & 0.449 \\
\hline Chronic pulmonary disease & $43(9.5)$ & $1(5.6)$ & $1.000 *$ \\
\hline Asthma & $39(8.6)$ & $2(11.1)$ & $0.665^{\star}$ \\
\hline Immunosuppressive state (other than malignancy) & $15(3.3)$ & $1(5.6)$ & $0.471^{*}$ \\
\hline Diabetes Mellitus & $75(16.6)$ & $3(16.7)$ & $1.000 *$ \\
\hline Renal disease & $45(10.0)$ & $3(16.7)$ & $0.414^{*}$ \\
\hline Neuromuscular disorders & $9(2.0)$ & $1(5.6)$ & $0.326^{*}$ \\
\hline Neurological disorders & $34(7.5)$ & $6(33.3)$ & $0.002^{*}$ \\
\hline Malignancy & $101(22.4)$ & $8(44.4)$ & $0.043^{*}$ \\
\hline Hemoglobinopathy & $15(3.3)$ & $0(0.0)$ & $1.000^{\star}$ \\
\hline Type of influenza & & & $1.000 *$ \\
\hline Influenza A & $363(80.5)$ & $15(83.3)$ & \\
\hline Influenza B & $79(17.5)$ & $3(16.7)$ & \\
\hline Co-infection $A \& B$ & $9(2.0)$ & $0(0.0)$ & \\
\hline Moderate to Severe disease $(\mathrm{N}=466)$ & $269(60.0)^{\dagger}$ & $18(100.0)$ & 0.001 \\
\hline
\end{tabular}




\begin{tabular}{|llll|} 
Radiologically confirmed pneumonia (N=418) & $173(43.3)^{\mathrm{a}}$ & $17(94.4)$ & $<0.001$ \\
\hline Proven viral co-infection & $13(2.9)$ & $7(38.9)$ & $<0.001$ * \\
\hline Proven bacterial co-infection & $94(20.8)$ & $11(61.1)$ & $\mathbf{0 . 0 0 1 *}$ \\
\hline
\end{tabular}

Pearson's Chi-Square test was used (no expected count less than 5).

* Fisher's exact test was used when expected count was less than 5

${ }^{\dagger} 3$ patients in this group had missing data

a 51 subjects had no imaging results

Table (3) shows risk factors for increased mortality among hospitalized patients due to influenza infection. A statistically significant P-value was found for moderate to severe disease, radiologically-confirmed pneumonia, and presence of viral or bacterial co-infection. Other statistically significant risk factors included neurological disorder (0.002) and malignancy (0.043). There was no statistically significant difference in mortality among different age groups or types of influenza infection detected. However, when stepwise logistic regression was done, only the presence of a proven viral co-infection, bacterial co-infection, underlying neurological diseases or radiologically-confirmed pneumonia were found to be independent risk factors for increased mortality (Table 4).

Table 4. Results of stepwise logistic regression for independent risk factors associated with increased mortality

\begin{tabular}{|lll|}
\hline Risk factors & ORa [95\% Cl] & p-value \\
\hline Neurological disorders & $4.2[1.3-13.9]$ & 0.018 \\
\hline Radiologically confirmed pneumonia & $13.7[1.7-108.1]$ & 0.013 \\
\hline Proven bacterial co-infection & $3.3[1.1-9.9]$ & 0.032 \\
\hline Proven viral co-infection & $11.4[3.3-40.1]$ & $<0.001$ \\
\hline
\end{tabular}

Included variables: Neurological disorders, malignancy, moderate to severe disease, radiologically confirmed pneumonia, proven bacterial co-infection, proven viral co-infection

\section{Antibiotic and antiviral use}

Antibiotics were prescribed for 494 subjects ( $41 \%$ of those with available data $N=1202)$ with laboratoryconfirmed influenza infection. Only $102(20.6 \%)$ of those who received antibiotics had a proven bacterial coinfection. A proven bacterial co-infection was considered in the presence of a positive culture. In fact, pneumonia cases accounted for $49 \%$ of antibiotic prescription though not all of these cases were proven to be bacterial. 
Antivirals were prescribed for 821 subjects ( $68.8 \%$ of those with available data $N=1193$ ) with laboratoryconfirmed influenza. Oseltamivir was prescribed in 807 subjects while zanamivir was prescribed in the remaining 14 subjects. Table (5) describes the different characteristics of the subjects who received antiviral treatment. The majority of elderly subjects and children below 2 years of age received antiviral therapy. There was a statistically significant higher percentage of antiviral prescription among inpatients, pregnant females and those with comorbid conditions.

Table 5. Characteristics of subjects receiving antiviral therapy over the study period.

\begin{tabular}{|c|c|c|}
\hline & Antiviral use, $\mathrm{n}(\%)$ & p-value \\
\hline Age groups & & $<0.001$ \\
\hline$[0-2$ years $[(N=120)$ & $97(80.8)$ & \\
\hline [2- 5 years $[(N=150)$ & $72(48.0)$ & \\
\hline$[5-10$ years $[(N=161)$ & $70(43.5)$ & \\
\hline$[10-19$ years $[(N=160)$ & $79(49.4)$ & \\
\hline [19- 50 years $[(N=296)$ & $219(74)$ & \\
\hline$[50-65$ years $[(N=144)$ & $129(89.6)$ & \\
\hline$\geq 65 \operatorname{years}(N=162)$ & $155(95.7)$ & \\
\hline Days since onset of symptoms & & 0.339 \\
\hline$\leq 2 \operatorname{days}(N=727)$ & $508(69.9)$ & \\
\hline$>2 \operatorname{days}(N=295)$ & $295(67.2)$ & \\
\hline \multicolumn{3}{|l|}{ Setting } \\
\hline Inpatients $(N=467)$ & $443(94.9)$ & $<0.001$ \\
\hline Outpatients $(N=725)$ & $378(52.1)$ & \\
\hline Comorbid conditions & & $<0.001$ \\
\hline Yes $(N=550)$ & $463(84.2)$ & \\
\hline No $(N=639)$ & $355(55.6)$ & \\
\hline Moderate to severe disease & & 0.059 \\
\hline Yes $(N=527)$ & $377(71.5)$ & \\
\hline No $(N=661)$ & $439(66.4)$ & \\
\hline Pregnant & & $0.003 *$ \\
\hline Yes $(N=64)$ & $54(84.4)$ & \\
\hline No $(N=169)$ & $108(63.9)$ & \\
\hline
\end{tabular}


Pearson's Chi-Square test was used (no expected count less than 5).

*Fisher's exact test was used when expected count was less than 5.

\section{Influenza type A, B and Co-infection}

Influenza detection was done using RDT or RT-PCR except for 2 cases where diagnosis was made by serology because the clinical picture was highly suggestive of influenza infection despite negative RDT and PCR. Most of the cases were diagnosed by RDT (1546 samples, $84.5 \%$ ) while 144 cases $(7.9 \%)$ were detected by PCR. In 137 cases (7.5\%), both RDT and PCR were used at the same time for detection. In these cases, PCR was likely requested for subtyping. Subtyping was done for 245 cases only, of which 138 cases were H1N1pdm09, 7 cases were H1N1 Brisbane, 61 cases were H3N2 and 3 cases were co-infected with $\mathrm{H} 1 \mathrm{~N} 1 \mathrm{pdm} 09$ and H3N2.As for influenza B cases, 20 were Victoria lineage, 14 were Yamagata and 2 were Shanghai lineage.

Characteristics of subjects with laboratory-confirmed influenza by virus type are illustrated in (Table 6). There was a significant variation in the incidence of each type of influenza virus through the different influenza seasons and among different age groups. Mean hospital stay duration was significantly higher in those who were co-infected with both types of influenza (17 days vs 7 days). Co-infection with both $A$ and $B$ viruses was significantly associated with higher incidence of ICU admission. Antibiotic and antiviral prescription was similar for both types and for co-infection. Death was equally reported for both types of influenza viruses $(P$-value $=1)$.

Table 6. Characteristics of laboratory confirmed influenza cases by virus type 


\begin{tabular}{|c|c|c|c|c|}
\hline Characteristics & $\begin{array}{l}\text { Influenza } A, \\
\mathrm{~N}=1529\end{array}$ & $\begin{array}{l}\text { Influenza } \mathrm{B} \text {, } \\
\mathrm{N}=242\end{array}$ & $\begin{array}{l}\text { Co-infection } \\
\text { A\&B, N=55 }\end{array}$ & p-value \\
\hline & n (\%) & n (\%) & $\mathrm{n}(\%)$ & \\
\hline Season $(N=1826)$ & & & & $<0.001^{*}$ \\
\hline Jan 2008-Jun 2008 & $12(66.7)$ & $6(33.3)$ & $0(0.0)$ & \\
\hline 2008-2009 & $47(78.3)$ & $6(10.0)$ & $7(11.7)$ & \\
\hline 2009-2010 & 529 (97.6) & $2(0.4)$ & $11(2.0)$ & \\
\hline $2010-2011$ & $43(58.9)$ & $25(34.2)$ & $5(6.8)$ & \\
\hline 2011-2012 & $92(84.4)$ & $2(1.8)$ & $15(13.8)$ & \\
\hline $2012-2013$ & $93(73.8)$ & $27(21.4)$ & $6(4.8)$ & \\
\hline 2013- 2014 & $183(89.3)$ & $17(8.3)$ & $5(2.4)$ & \\
\hline 2014- 2015 & $153(64.3)$ & $82(34.5)$ & $3(1.3)$ & \\
\hline 2015-2016 & 377 (82.9) & $75(16.5)$ & $3(0.7)$ & \\
\hline \multicolumn{5}{|l|}{ Age group $(\mathrm{N}=1826)$} \\
\hline$[0-2$ years [ & $134(8.8)$ & $28(11.6)$ & $11(20.0)$ & $0.039^{¥}$ \\
\hline [2- 5 years [ & $205(13.4)$ & $25(10.3)$ & $9(16.4)$ & \\
\hline [5-10 years [ & $289(18.9)$ & $44(18.2)$ & $5(9.1)$ & \\
\hline [10-19 years [ & $283(18.5)$ & $37(15.3)$ & $7(12.7)$ & \\
\hline [19- 50 years [ & $340(22.2)$ & $56(23.1)$ & $14(25.5)$ & \\
\hline [50-65 years [ & $138(9.0)$ & $25(10.3)$ & $1(1.8)$ & \\
\hline$\geq 65$ years & $140(9.2)$ & $27(11.2)$ & $8(14.5)$ & \\
\hline Gender $(N=1826)$ & & & & 0.992 \\
\hline Male & $764(50.0)$ & $121(50.0)$ & $27(49.1)$ & \\
\hline Female & $765(50.0)$ & $121(50.0)$ & $28(50.9)$ & \\
\hline \multicolumn{5}{|l|}{$\begin{array}{l}\text { Underlying medical conditions } \\
(\mathrm{N}=1300)\end{array}$} \\
\hline Any & $511(47.8)$ & $89(45.2)$ & $15(44.1)$ & 0.74 \\
\hline Cardiovascular disease & $179(16.7)$ & $35(17.8)$ & $8(23.5)$ & 0.563 \\
\hline Chronic pulmonary disease & $51(4.8)$ & $3(1.5)$ & $0(0.0)$ & 0.054 \\
\hline Asthma & $135(12.6)$ & $20(10.2)$ & $5(14.7)$ & 0.543 \\
\hline Immunosuppressive state (other than & $16(1.5)$ & $3(1.5)$ & $0(0.0)$ & $1.000 *$ \\
\hline
\end{tabular}




\begin{tabular}{|c|c|c|c|c|}
\hline Diabetes Mellitus & $81(7.6)$ & $13(6.6)$ & $2(5.9)$ & $0.938 *$ \\
\hline Renal disease & $43(4.0)$ & $8(4.1)$ & $1(2.9)$ & $1.000 *$ \\
\hline Neuromuscular disorders & $8(0.7)$ & $4(2.0)$ & $0(0.0)$ & $0.204^{*}$ \\
\hline Neurological disorders & $47(4.4)$ & $8(4.1)$ & $3(8.8)$ & $0.395^{\star}$ \\
\hline Malignancy & $136(12.7)$ & $27(13.7)$ & $1(2.9)$ & $0.213^{*}$ \\
\hline Hemoglobinopathy & $27(2.5)$ & $3(1.5)$ & $0(0.0)$ & $0.749 *$ \\
\hline Antibiotic prescription ( $\mathrm{N}=1201)$ & $395(40.0)$ & $87(47.8)$ & $12(37.5)$ & 0.134 \\
\hline Antiviral prescription ( $\mathrm{N}=1191)$ & $684(69.8)$ & $119(65.7)$ & $17(56.7)$ & 0.192 \\
\hline Hospitalization rate $(\mathrm{N}=1784)$ & $381(25.4)$ & $84(36.2)$ & $10(18.5)$ & 0.001 \\
\hline \multirow[t]{2}{*}{ Characteristics } & $\begin{array}{l}\text { Influenza A, } \\
\mathrm{N}=381\end{array}$ & $\begin{array}{l}\text { Influenza B, } \\
\mathrm{N}=84\end{array}$ & $\begin{array}{l}\text { Co-infection } \\
\mathrm{A} \& \mathrm{~B}, \mathrm{~N}=10\end{array}$ & p-value \\
\hline & $\mathrm{n}(\%)$ & $\mathrm{n}(\%)$ & $\mathrm{n}(\%)$ & \\
\hline \multicolumn{5}{|l|}{$\begin{array}{l}\text { Progression of illness and in-hospital } \\
\text { outcome }\end{array}$} \\
\hline Moderate to severe disease $(N=470)$ & $224(59.4)$ & $59(71.1)$ & $7(70.0)$ & $0.129 *$ \\
\hline ICU admission $(N=475)$ & $51(13.4)$ & $15(17.9)$ & $4(40.0)$ & $0.042^{*}$ \\
\hline Oxygen therapy $(N=470)$ & $87(23.0)$ & $20(24.4)$ & $4(40.0)$ & $0.399 *$ \\
\hline \multirow[t]{2}{*}{ Mortality $(N=470)$} & $15(4.0)$ & $3(3.7)$ & $0(0.0)$ & $1.000 *$ \\
\hline & Mean ( \pm SD) & Mean ( \pm SD) & Mean ( \pm SD) & p-value \\
\hline Hospital stay duration $(\mathrm{N}=473)^{\pi}$ & $6.8( \pm 8.4)$ & $6.8( \pm 6.7)$ & $16.7( \pm 23.8)$ & 0.02 \\
\hline
\end{tabular}

Percentages are expressed within each influenza type, except for the season variable in which percentages are expressed within each season

Pearson's Chi-Square test was used (no expected count less than 5).

Independent Sample-t test is used to compare means.

${ }^{*}$ Fisher's exact test was used when expected count was less than 5.

¥ Monte Carlo estimate was used when the data set was too large to compute exact significance

$\pi$ Two patients were excluded; one stayed in 113 days and the other stayed for 262 days related to their underlying medical condition (Neuromuscular disease and cerebral palsy) 
Figure (1) shows the monthly distribution of influenza A and B through the various seasons. Influenza A was mostly detected during the months of January (37.4\%) and February (34.8\%). Another peak, however, is seen in November (51.2\%) mostly during the 2009 pandemic. Influenza B tended to peak later during the month of March (32.1\%). Over the different seasons, influenza infections started by the month of November and peaked by January except during the 2009 pandemic when a first wave was seen during the summer months and peaked in August and then another significant wave that started in October and peaked in November.

\section{Influenza hospitalization rates}

Overall influenza-associated hospitalization rate reached $26.6 \%$. The annual influenza-associated hospitalization rate was lowest during the 2009 pandemic season (6.8\%) and trended up gradually to reach the highest during 2016 (41\%) (Figure 2). In addition, the monthly influenza-associated hospitalization rate was as low as $3 \%$ in July and reached almost $37 \%$ during January (Figure 3 ).

\section{Discussion}

Our results show that seasonal influenza causes a substantial burden in the Lebanese population. The AUBMC serves an annual average of around 400,000 persons including in-hospital admissions, clinic visits and ED visits. A total of 11288 subjects were tested for influenza virus during the study period that extended over 8 influenza seasons of which 1829 (16\%) subjects were positive for either influenza A, B or both. A total of $475(26.6 \%)$ subjects were hospitalized. This means that an annual average of 200 subjects served by the medical center had a confirmed influenza infection and accounted for $0.017 \%$ of all-cause yearly hospital visits. This number is lower than what was reported by Saleh et al in their surveillance data, which reached $0.2 \%$ at their sentinel site in Beirut. (35) This is explained by the larger denominator, which, unlike SARI surveillance reported by Saleh et al, also included both ED visits and ambulatory same day services. We could not narrow down the denominator to visits due to respiratory illnesses only instead of all-cause hospital visits for several reasons but mainly because our retrospective data included any subject tested for influenza even if they did not fit the ILI or SARI definition and thus many may have sought medical care for non-respiratory complaints. The average annual positivity rate for tested subjects was $14 \%$ and this is similar to the positivity rate (13.4\%) reported by the Lebanese SARI Sentinel Surveillance conducted by the Ministry of Public Health for the 2015/2016 season.(35) Moghoofei et al estimated an influenza infection prevalence of $10 \%$ in the Middle East that approaches our positivity rate in Lebanon.(36) In his systematic review, 71 studies were included of which only one study was from Lebanon but included a very small sample size.(30)

According to the WHO Global Epidemiological Surveillance Standards for Influenza, a standard age grouping into 6 groups is suggested for analysis (5). We further subdivided the population into an additional 7th 'adolescent' age group (10-19 years). Results from the SARI surveillance by the MOH showed a similar distribution, with higher positivity rates among those $15-49$ years reaching $26 \%$ in the southern sentinel site and a positivity rate of $21 \%$ for those $5-15$ years in the Beirut sentinel site. (35) In addition to surveying 
sicker hospitalized subjects, an additional explanation may be that these age groups are less likely to be vaccinated when compared to the very young and the very old. According to a cross-sectional review published in 2015, influenza vaccine uptake among Lebanese adults was only $27.6 \%$ during the 2014/2015 season.(37) In Lebanon, influenza vaccination is only recommended for subjects with a chronic medical condition, hospital healthcare workers and pilgrims.(38) The vaccination rate for our population reached $22.4 \%$. Unfortunately, we did not have vaccination data for most of our subjects and the majority of available data was for pediatric patients. It is important to recall that although influenza vaccines were thought to be mostly effective for children 2 years and older, recent data showed high vaccine efficacy reaching $60 \%$ in infants as young as 6 months $(34,39-43)$.

Our analysis showed that all severity parameters, except for hospital and ICU stay duration, were highly present within two age groups: below 2 years and $\geq 65$ years. These results demonstrate that extremes of age have a more severe course of disease including a higher mortality rate. Several recommending bodies consider these age groups at higher risk for severe disease and mortality and thus recommend early initiation of antiviral therapy and emphasize the importance of vaccination. $(44,45)$ Unfortunately, vaccination is not recommended for these age groups nor included in the national immunization program in Lebanon. (38)

The presence of at least one co-morbidity, mainly cardiovascular diseases, asthma, malignancy and diabetes mellitus, in $71 \%$ of hospitalized patients and $31 \%$ of outpatients is worrisome especially with recent data on influenza being a trigger for exacerbation of these illnesses and vice versa. Several papers have showed an association between influenza infection and myocardial infarction, chronic obstructive pulmonary disease exacerbations and diabetic crises. $(18,23,24,27,46,47)$ This also means that at least half of the study population was at higher risk for severe influenza disease and outcome including death.

Several complications have been linked to influenza infection including both pulmonary and extrapulmonary. Both bacterial and viral pneumonias are established complications of influenza infection and have been reported as an independent risk factor for increased severity and mortality. (49-51) Similarly, our review showed that radiologically confirmed pneumonia was an independent risk factor for increased mortality among hospitalized patients (Table 4). It was the most common complication reported with highest incidence in older age groups $>50$ years. We could not determine whether these were primary viral pneumonias or bacterial co-infection in most of the cases. Inflammatory markers including C-reactive protein and procalcitonin were not used regularly especially in outpatients, so we did not track them. Regardless, $89 \%$ of these cases received antimicrobial treatment according to the treating physician's judgment. Of these pneumonia cases, 16 (6.6\%) progressed to acute respiratory distress syndrome (ARDS).

Our population also displayed a wide array of extra-pulmonary complications including myocardial infarction, encephalopathy, shock, renal failure and liver failure. These complications have been reported in several articles (52-57) and interest in determining their real burden is increasing as these extra-pulmonary complications actually represent a significant burden related to influenza infection that is sometimes overlooked.(53) 
According to the global burden of diseases study, influenza accounted for $0.26 \%$ of all-cause mortality globally. It also estimated that 0.6 per 100,000 population died from influenza in Lebanon.(3) In our review, we identified 18 fatalities representing $3.8 \%$ of all hospitalized laboratory proven influenza cases during an 8-year period. Unfortunately, national mortality data is lacking. In Egypt, according to influenza surveillance data from 2007 to 2014 , influenza positivity was $17 \%$ among SARI cases with a mortality rate reaching $2 \%$. (58) In Oman, influenza-associated deaths from 2012-2015 were estimated at 0.9 per 100,000 population and was highest among those $\geq 65$ years (18.6 per 100,000 population).(59) This was similarly reported by the Global Burden of Diseases study where the population attributable fraction (PAF) for adults older than 70 years was $6.3 \%$ compared to $2.9 \%$ for children younger than 5 years. (3) This is similar to our data where the highest mortality was in those $\geq 65$ years (3.6\%) compared to $1.5 \%$ in those below 2 years. According to our results, factors found to be associated with increased mortality were underlying neurological disease, malignancy, moderate to severe disease, proven viral or bacterial co-infection, and presence of pneumonia (P-value $<0.05)$. However, after multivariate logistic regression, only the presence of a proven viral or bacterial co-infection, pneumonia and neurological disease were independent risk factors for increased mortality. Unlike the data from Egypt (58), we didn't find any association between influenza type and mortality. A systematic review by Klein et al showed a very variable range in the incidence of bacterial coinfection with influenza from $2 \%$ to $65 \%$ in different studies.(60) It is however associated with an increased incidence of morbidity and mortality in all age groups for both seasonal and pandemic influenza with Staphylococcus aureus and Streptococcus pneumoniae being the most common pathogens identified. (60, 61) With the advance in the detection methods used to identify causative viral pathogens, several papers have discussed the increasing incidence of viral co-infection and its role in the severity of influenza infection. $(62,63)$ Though our results did show a higher rate of viral co-infection in the mortality cases, most of these viruses were not respiratory viruses. With the advent of routine use of a multiplex PCR respiratory panel at many centers around the world, more cases of co-infection with other respiratory viruses are likely to be detected. Also similar to our results, underlying chronic medical conditions have been reported as a risk factor for mortality $(48,58,64)$ and neurological disease has been found to be an independent risk factor. (48)

According to the recent guidelines by the Infectious Diseases Society of America (IDSA), antiviral treatment should be started when influenza infection is suspected or confirmed in any hospitalized patient, those with severe or progressive illness, in children $\leq 2$ years and adults $\geq 65$ years old, pregnant females and 2 weeks postpartum, extremely obese patients, children and adolescents receiving salicylate-containing medications, patients with chronic medical conditions including immunosuppression and residents of chronic care centers. (45) Treatment should also be considered for contacts of high-risk patients mentioned above (household or healthcare providers) and when symptoms' onset is $\leq 2$ days at presentation. When treatment is warranted, a single neuraminidase inhibitor (NAl) should be used. $(45,65)$ Oseltamivir was almost exclusively used in our population. It was prescribed for almost $70 \%$ of the subjects. Our review period, however, includes the 2009 pandemic when NAl's were used extensively; but even when excluded in the analysis, the rate of antiviral use remains high (73\%). Our data shows that there was a tendency to follow the guidelines though maybe not completely. Antiviral prescription was significantly higher at the extremes of age reaching $96 \%$ in $\geq 65$ years, $95 \%$ of hospitalized subjects, $84 \%$ of subjects with underlying high-risk 
conditions and $84 \%$ of pregnant females. Nonetheless, it seems that the duration since onset of symptoms and the severity of disease per se did not influence the decision to initiate antivirals. A more detailed description of each case would further evaluate the level of adherence to the guidelines in order to implement a judicious plan for the use of these antivirals and try to control influenza resistance. On the other hand, prompt use of antivirals may reduce unnecessary use of antibiotics by decreasing the risk of bacterial co-infections, which are reported to be as low as $2 \% .(45,66,67)$ This implies the need for a more thorough evaluation before the decision to start antibiotics due to the increasing reports of resistant organisms mainly MRSA. $(60,68)$ Nonetheless, recommendations provided by the IDSA emphasize the use of antibiotics empirically in severe cases of influenza and in those who deteriorate after initial improvement. (45) Antibiotics were prescribed in around $40 \%$ of subjects, half of which had an underlying pneumonia. A similar rate of antibiotic prescription was reported in Algeria (41\%) (69) and Switzerland (45\%) (70).

Both influenza A and B co-circulated during the 8 seasons with influenza $A$ being predominant during each season. Historically, there has been more interest in researching influenza A virus mainly because of its capability to cause pandemics and underestimating the burden of influenza B virus. However, our data shows an increased morbidity associated with influenza B evident mainly by the higher incidence of ICU admissions although with no difference in mortality incidence for both types. Earlier published data have shown an increasing burden for influenza B in the USA, (71) whereas data from other countries showed a contradicting role of type $B$ in influenza related morbidity and mortality. $(58,71-74)$ Further populationbased studies are needed to look further into the real burden of Influenza B and comparing its clinical characteristics to influenza A.

In the MENA region, there is limited data on the circulation patterns of both influenza A and B. It is of paramount importance to determine the timing of influenza circulation for each region in order to decide on the best timing for vaccine delivery, aid healthcare workers in their clinical management of suspected cases in addition to increasing emphasis on prevention of infection and control of transmission. (75) In his review of surveillance data from the MENA region for the time period 2010-2016, Caini et al. reported that most of the region's countries had a primary peak between January and March with no or barely noticeable secondary peaks. (76) According to the Lebanese SARI sentinel surveillance, the primary peak extended from January till mid of March.(35) This is consistent with our data, which shows that the influenza season starts by November-December and peaks during January and February. These results imply that vaccination should be administered between September and October of each year to ensure optimal protection.

Our study has several limitations. The most significant limitation is the fact that this data was collected retrospectively from chart review. We could not obtain accurate data on vaccination rates in addition to other missing or incomplete data. Another limitation is that all these cases reviewed were from a single center that, despite being a major tertiary medical center, cannot reflect the actual Lebanese burden of influenza. A third limitation is the fact that we included all laboratory proven influenza cases regardless of initial presentation symptoms or reason for admission. As a result, we could not calculate influenza-attributable SARI cases and we could not compare this to other published data. Also, we did not exclude possible healthcare associated infections, although they contribute to the burden of influenza. Moreover, mortality rate 
associated with influenza might not be accurate since we had no follow up data on outpatients and thus significant data was missing.

\section{Conclusion}

This study is a first attempt to estimate the burden of influenza in Lebanon including a large number of subjects. Despite being a retrospective review, results offer groundwork for further influenza research. Based on the results presented by our review, influenza causes a substantial number of hospitalizations annually in Lebanon with significant morbidity and mortality across all age groups. Even though we had incomplete data on vaccination, a rate of $22 \%$ is fairly low. Fortunately, there is an increased interest in influenza research and surveillance along with increased testing for ILI and SARI cases. The SARI sentinel surveillance initiated by the Ministry of Public Health since 2015 promises to provide better data on the burden of influenza on a national level thus aid in developing a better vaccination program and maybe incorporate it into the national immunization program. A population based prospective surveillance study is needed to better estimate the burden of influenza in Lebanon which would help to drive policy on influenza control.

\section{Abbreviations}

WHO: World Health Organization; MENA: Middle East and North Africa; ILI: influenza-like illness; SARI: Severe Acute Respiratory Infection; AUBMC: American University of Beirut Medical Center; CIDR: Center for Infectious Diseases Research; RDT: Rapid Diagnosis Test; PCR: Polymerase Chain Reaction; CRF: Case Report Form; CT: Computed Tomography.

\section{Declarations}

\section{Acknowledgement}

The authors thank Bassel Hafez and Joudie Alwan for their contribution in record review.

Funding sources: This research did not receive any specific grant from funding agencies in the public, commercial, or not-for-profit sectors.

\section{Authors Contributions:}

AA, HZ, RHW and GD designed the study. AA, ZS, SF, HE, MS, SC, RS, SLS, AC collected data. SK performed statistical analysis and participated in the results writing. AA wrote the initial draft of manuscript. RM and GA conducted the laboratory assays. SSK, ZK, RHW, RS, HS, HZ, and GD critically revised the manuscript. All authors read and approved the final version of the manuscript.

Availability of data and materials: The datasets used and/or analysed during the current study are available from the corresponding author on reasonable request.

Ethics approval: Ethical clearance was obtained from the Institutional Review Board at AUBMC (PED.GD.11) 
Consent for publication : Not applicable.

Competing interests: The authors declare that they have no competing interests

\section{References}

1.Ting SC, Crooks SW, South G. The effect of influenza vaccination on the incidence of chronic obstructive pulmonary disease exacerbations in the immediate postvaccination period. J Epidemiol Community Health. 2011;65(2):157-9. DOI: 10.1136/jech.2009.092296.

2.Simoes EAF, Cherian T, Chow J, Shahid-Salles SA, Laxminarayan R, John TJ. Acute Respiratory Infections in Children. In: nd, Jamison DT, Breman JG, Measham AR, Alleyne G, Claeson M, et al., editors. Disease Control Priorities in Developing Countries. Washington (DC)2006.

3.Collaborators GBDI. Mortality, morbidity, and hospitalisations due to influenza lower respiratory tract infections, 2017: an analysis for the Global Burden of Disease Study 2017. Lancet Respir Med. 2019;7(1):69-89. DOI: 10.1016/S2213-2600(18)30496-X.

4.Meerhoff TJ, Simaku A, Ulqinaku D, Torosyan L, Gribkova N, Shimanovich V, et al. Surveillance for severe acute respiratory infections (SARI) in hospitals in the WHO European region - an exploratory analysis of risk factors for a severe outcome in influenza-positive SARI cases. BMC Infect Dis. 2015;15:1. DOI: 10.1186/s12879-014-0722-x.

5.Organization WH. Global Epidemiological Surveillance Standards for Influenza. Geneva, Switzerland: World Health Organization; 2013. 73 p.

6.Zimmerman RK, Rinaldo CR, Nowalk MP, Gk B, Thompson MG, Moehling KK, et al. Influenza and other respiratory virus infections in outpatients with medically attended acute respiratory infection during the 2011-12 influenza season. Influenza Other Respir Viruses. 2014;8(4):397-405. DOI: 10.1111/irv.12247.

7.Katz MA, Muthoka P, Emukule GO, Kalani R, Njuguna H, Waiboci LW, et al. Results from the first six years of national sentinel surveillance for influenza in Kenya, July 2007-June 2013. PLoS One. 2014;9(6):e98615. DOI: 10.1371/journal.pone.0098615.

8.Huang QS, Baker M, McArthur C, Roberts S, Williamson D, Grant C, et al. Implementing hospital-based surveillance for severe acute respiratory infections caused by influenza and other respiratory pathogens in New Zealand. Western Pac Surveill Response J. 2014;5(2):23-30. DOI: 10.5365/WPSAR.2014.5.1.004.

9.Azziz-Baumgartner E, Alamgir AS, Rahman M, Homaira N, Sohel BM, Sharker MA, et al. Incidence of influenza-like illness and severe acute respiratory infection during three influenza seasons in Bangladesh, 2008-2010. Bull World Health Organ. 2012;90(1):12-9. DOI: 10.2471/BLT.11.090209.

10.Lau LL, Cowling BJ, Fang VJ, Chan KH, Lau EH, Lipsitch M, et al. Viral shedding and clinical illness in naturally acquired influenza virus infections. J Infect Dis. 2010;201(10):1509-16. DOI: 10.1086/652241. 
11. Hayward AC, Fragaszy EB, Bermingham A, Wang L, Copas A, Edmunds WJ, et al. Comparative community burden and severity of seasonal and pandemic influenza: results of the Flu Watch cohort study. Lancet Respir Med. 2014;2(6):445-54. DOI: 10.1016/S2213-2600(14)70034-7.

12.Hsieh Y-H, Tsai C-A, Lin C-Y, Chen J-H, King C-C, Chao D-Y, et al. Asymptomatic ratio for seasonal H1N1 influenza infection among schoolchildren in Taiwan. BMC Infectious Diseases. 2014;14(1):1-10. DOI: 10.1186/1471-2334-14-80.

13.Rello J, Rodriguez A, Ibanez P, Socias L, Cebrian J, Marques A, et al. Intensive care adult patients with severe respiratory failure caused by Influenza A (H1N1)v in Spain. Crit Care. 2009;13(5):R148. DOI: $10.1186 /$ cc8044.

14.Rothberg MB, Haessler SD, Brown RB. Complications of viral influenza. Am J Med. 2008;121(4):258-64. DOI: 10.1016/j.amjmed.2007.10.040.

15.Guarner J, Paddock CD, Shieh WJ, Packard MM, Patel M, Montague JL, et al. Histopathologic and immunohistochemical features of fatal influenza virus infection in children during the 2003-2004 season. Clin Infect Dis. 2006;43(2):132-40. DOI: 10.1086/505122.

16.Grohskopf LA, Sokolow LZ, Olsen SJ, Bresee JS, Broder KR, Karron RA. Prevention and Control of Influenza With Vaccines: Recommendations of the Advisory Committee on Immunization Practices, United States, 2015-16 Influenza Season. Am J Transplant. 2015;15(10):2767-75. DOI: 10.1111/ajt.13505.

17.Vaccines against influenza WHO position paper - November 2012. Wkly Epidemiol Rec. 2012;87(47):46176.

18.Kwong JC, Schwartz KL, Campitelli MA, Chung H, Crowcroft NS, Karnauchow T, et al. Acute Myocardial Infarction after Laboratory-Confirmed Influenza Infection. N Engl J Med. 2018;378(4):345-53. DOI: 10.1056/NEJMoa1702090.

19.Ludwig A, Lucero-Obusan C, Schirmer P, Winston C, Holodniy M. Acute cardiac injury events $</=30$ days after laboratory-confirmed influenza virus infection among U.S. veterans, 2010-2012. BMC Cardiovasc Disord. 2015;15:109. DOI: 10.1186/s12872-015-0095-0.

20.Chiang MH, Wu HH, Shih CJ, Chen YT, Kuo SC, Chen TL. Association between influenza vaccination and reduced risks of major adverse cardiovascular events in elderly patients. Am Heart J. 2017;193:1-7. DOI: 10.1016/j.ahj.2017.07.020.

21.Geladari E, Papademetriou V, Moore H, Lu D. A case of influenza type a myocarditis that presents with ST elevation $\mathrm{Ml}$, cardiogenic shock, acute renal failure, and rhabdomyolysis and with rapid recovery after treatment with oseltamivir and intra-aortic balloon pump support. Cardiovasc Revasc Med. 2018;19(1 Pt A):37-42. DOI: 10.1016/j.carrev.2017.04.017.

22.Collins JP, Campbell AP, Openo K, Farley MM, Cummings CN, Kirley PD, et al. Clinical Features and Outcomes of Immunocompromised Children Hospitalized With Laboratory-Confirmed Influenza in the United 
States, 2011-2015. J Pediatric Infect Dis Soc. 2018. DOI: 10.1093/jpids/piy101.

23.Panhwar MS, Kalra A, Gupta T, Kolte D, Khera S, Bhatt DL, et al. Effect of Influenza on Outcomes in Patients With Heart Failure. JACC Heart Fail. 2019;7(2):112-7. DOI: 10.1016/j.jchf.2018.10.011.

24. Homaira N, Briggs N, Oei JL, Hilder L, Bajuk B, Snelling T, et al. Impact of influenza on hospitalization rates in children with a range of chronic lung diseases. Influenza Other Respir Viruses. 2019. DOI: 10.1111/irv.12633.

25.Pearce DC, McCaw JM, McVernon J, Mathews JD. Influenza as a trigger for cardiovascular disease: An investigation of serotype, subtype and geographic location. Environ Res. 2017;156:688-96. DOI: 10.1016/j.envres.2017.04.024.

26.Huang HH, Chen SJ, Chao TF, Liu CJ, Chen TJ, Chou P, et al. Influenza vaccination and risk of respiratory failure in patients with chronic obstructive pulmonary disease: A nationwide population-based case-cohort study. J Microbiol Immunol Infect. 2019;52(1):22-9. DOI: 10.1016/j.jmii.2017.08.014.

27.Hulme KD, Gallo LA, Short KR. Influenza Virus and Glycemic Variability in Diabetes: A Killer Combination? Front Microbiol. 2017;8:861. DOI: 10.3389/fmicb.2017.00861.

28.Abusrewil S, Algeer A, Aljifri A, Al Slail F, Andrew MK, Awad Tag Eldin M, et al. Influenza surveillance in Middle East, North, East and South Africa: Report of the 8th MENA Influenza Stakeholders Network. Influenza Other Respir Viruses. 2019. DOI: 10.1111/irv.12628.

29.Zaraket H, Dapat C, Ghanem S, Ali Z, Lteif M, Kondo H, et al. Characterization of human Influenza Viruses in Lebanon during 2010-2011 and 2011-2012 post-pandemic seasons. Intervirology. 2014;57(6):344-52. DOI: $10.1159 / 000365758$.

30.Zaraket H, Dbaibo G, Salam O, Saito R, Suzuki H. Influenza virus infections in Lebanese children in the 2007-2008 season. Jpn J Infect Dis. 2009;62(2):137-8.

31.Zaraket H, Kondo H, Tabet C, Hanna-Wakim R, Suzuki Y, Dbaibo GS, et al. Genetic diversity and antiviral drug resistance of pandemic H1N1 2009 in Lebanon. J Clin Virol. 2011;51(3):170-4. DOI:

10.1016/j.jcv.2011.04.001.

32.Zaraket H, Saito R, Wakim R, Tabet C, Medlej F, Reda M, et al. Antiviral drug susceptibilities of seasonal human influenza viruses in Lebanon, 2008-09 season. J Med Virol. 2010;82(7):1224-8. DOI:

10.1002/jmv.21795.

33.Saito R AH, Shaker RA, Akel IS, Assaf-Casals A, Lteif M, Odagiri T, Inaba R, Soudani N, Khafaja S, Ghanem ST, Rajab M, Shobugawa Y, Dbaibo GS, Zaraket H. Characterization of influenza outbreaks in Lebanon during the 2013/14 and 2014/15 seasons. East Mediterr Health J 2016;22(7):547-51.

34.Claeys C, Zaman K, Dbaibo G, Li P, Izu A, Kosalaraksa P, et al. Prevention of vaccine-matched and mismatched influenza in children aged 6-35 months: a multinational randomised trial across five influenza 
seasons. Lancet Child Adolesc Health. 2018;2(5):338-49. DOI: 10.1016/S2352-4642(18)30062-2.

35.Saleh M, Bazzi L, Ismail E, Mroueh L, Jammal N, Elkholy A, et al. Influenza-associated severe acute respiratory infections in 2 sentinel sites in Lebanon-September 2015 to August 2016. Influenza Other Respir Viruses. 2018;12(3):331-5. DOI: 10.1111/irv.12527.

36.Moghoofei M, Monavari SH, Mostafaei S, Hadifar S, Ghasemi A, Babaei F, et al. Prevalence of influenza A infection in the Middle-East: A systematic review and meta-analysis. Clin Respir J. 2018;12(5):1787-801. DOI: $10.1111 /$ crj.12758.

37.Zaraket H, Melhem N, Malik M, Khan WM, Dbaibo G, Abubakar A. Review of seasonal influenza vaccination in the Eastern Mediterranean Region: Policies, use and barriers. J Infect Public Health. 2018. DOI: 10.1016/j.jiph.2018.10.009.

38.Abubakar A, Melhem N, Malik M, Dbaibo G, Khan WM, Zaraket H. Seasonal influenza vaccination policies in the Eastern Mediterranean Region: Current status and the way forward. Vaccine. 2019;37(12):1601-7. DOI: $10.1016 /$ j.vaccine.2019.02.001.

39.Belongia EA, Kieke BA, Donahue JG, Greenlee RT, Balish A, Foust A, et al. Effectiveness of inactivated influenza vaccines varied substantially with antigenic match from the 2004-2005 season to the 2006-2007 season. J Infect Dis. 2009;199(2):159-67. DOI: 10.1086/595861.

40.Flannery B, Clippard J, Zimmerman RK, Nowalk MP, Jackson ML, Jackson LA, et al. Early estimates of seasonal influenza vaccine effectiveness - United States, January 2015. MMWR Morb Mortal Wkly Rep. 2015;64(1):10-5.

41.Jain VK, Rivera L, Zaman K, Espos RA, Jr., Sirivichayakul C, Quiambao BP, et al. Vaccine for prevention of mild and moderate-to-severe influenza in children. N Engl J Med. 2013;369(26):2481-91. DOI: 10.1056/NEJMoa1215817.

42.Jefferson T, Rivetti A, Di Pietrantonj C, Demicheli V. Vaccines for preventing influenza in healthy children. Cochrane Database Syst Rev. 2018;2:CD004879. DOI: 10.1002/14651858.CD004879.pub5.

43.McLean HQ, Thompson MG, Sundaram ME, Kieke BA, Gaglani M, Murthy K, et al. Influenza vaccine effectiveness in the United States during 2012-2013: variable protection by age and virus type. J Infect Dis. 2015;211(10):1529-40. DOI: 10.1093/infdis/jiu647.

44.Committee On Infectious D. Recommendations for Prevention and Control of Influenza in Children, 20182019. Pediatrics. 2018;142(4). DOI: 10.1542/peds.2018-2367.

45.Uyeki TM, Bernstein HH, Bradley JS, Englund JA, File TM, Fry AM, et al. Clinical Practice Guidelines by the Infectious Diseases Society of America: 2018 Update on Diagnosis, Treatment, Chemoprophylaxis, and Institutional Outbreak Management of Seasonal Influenzaa. Clin Infect Dis. 2019;68(6):e1-e47. DOI: $10.1093 /$ cid/ciy866. 
46.Ruiz PLD, Tapia G, Bakken IJ, Haberg SE, Hungnes O, Gulseth HL, et al. Pandemic influenza and subsequent risk of type 1 diabetes: a nationwide cohort study. Diabetologia. 2018;61(9):1996-2004. DOI: $10.1007 / \mathrm{s} 00125-018-4662-7$.

47.Capua I, Mercalli A, Romero-Tejeda A, Pizzuto MS, Kasloff S, Sordi V, et al. Study of 2009 H1N1 Pandemic Influenza Virus as a Possible Causative Agent of Diabetes. J Clin Endocrinol Metab. 2018;103(12):4343-56. DOI: 10.1210/jc.2018-00862.

48.Coleman BL, Fadel SA, Fitzpatrick T, Thomas SM. Risk factors for serious outcomes associated with influenza illness in high- versus low- and middle-income countries: Systematic literature review and metaanalysis. Influenza Other Respir Viruses. 2018;12(1):22-9. DOI: 10.1111/irv.12504.

49.Daoud A, Laktineh A, Macrander C, Mushtaq A, Soubani AO. Pulmonary complications of influenza infection: a targeted narrative review. Postgrad Med. 2019:1-10. DOI: 10.1080/00325481.2019.1592400.

50.Ishiguro T, Kagiyama N, Uozumi R, Odashima K, Takaku Y, Kurashima K, et al. Clinical Characteristics of Influenza-Associated Pneumonia of Adults: Clinical Features and Factors Contributing to Severity and Mortality. Yale J Biol Med. 2017;90(2):165-81.

51.Metersky ML, Masterton RG, Lode H, File TM, Jr., Babinchak T. Epidemiology, microbiology, and treatment considerations for bacterial pneumonia complicating influenza. Int J Infect Dis. 2012;16(5):e321-31. DOI: 10.1016/j.jijid.2012.01.003.

52.Steininger C, Popow-Kraupp T, Laferl H, Seiser A, Godl I, Djamshidian S, et al. Acute encephalopathy associated with influenza A virus infection. Clin Infect Dis. 2003;36(5):567-74. DOI: 10.1086/367623.

53.Sellers SA, Hagan RS, Hayden FG, Fischer WA, 2nd. The hidden burden of influenza: A review of the extrapulmonary complications of influenza infection. Influenza Other Respir Viruses. 2017;11(5):372-93. DOI: 10.1111/irv.12470.

54.Naderi ASA, Palmer BF. Rhabdomyolysis and acute renal failure associated with influenza virus type B infection. Am J Med Sci. 2006;332(2):88-9. DOI: 10.1097/00000441-200608000-00007.

55.Morishima T, Togashi T, Yokota S, Okuno Y, Miyazaki C, Tashiro M, et al. Encephalitis and encephalopathy associated with an influenza epidemic in Japan. Clin Infect Dis. 2002;35(5):512-7. DOI: 10.1086/341407.

56.Barnes M, Heywood AE, Mahimbo A, Rahman B, Newall AT, Macintyre CR. Acute myocardial infarction and influenza: a meta-analysis of case-control studies. Heart. 2015;101(21):1738-47. DOI:

10.1136/heartjnl-2015-307691.

57.Annerstedt M, Herlitz H, Molne J, Oldfors A, Westberg G. Rhabdomyolysis and acute renal failure associated with influenza virus type A. Scand J Urol Nephrol. 1999;33(4):260-4.

58.Kandeel A, Dawson P, Labib M, Said M, El-Refai S, El-Gohari A, et al. Morbidity, Mortality, and Seasonality of Influenza Hospitalizations in Egypt, November 2007-November 2014. PLoS One. 2016;11(9):e0161301. 
DOI: 10.1371/journal.pone.0161301.

59.Abdel-Hady DM, Al Balushi RM, Al Abri BA, Al Abri SS, Al Kindi HS, Al-Jardani AK, et al. Estimating the burden of influenza-associated hospitalization and deaths in Oman (2012-2015). Influenza Other Respir Viruses. 2018;12(1):146-52. DOI: 10.1111/irv.12500.

60.Klein EY, Monteforte B, Gupta A, Jiang W, May L, Hsieh YH, et al. The frequency of influenza and bacterial coinfection: a systematic review and meta-analysis. Influenza Other Respir Viruses. 2016;10(5):394-403. DOI: $10.1111 /$ irv. 12398.

61.Joseph C, Togawa Y, Shindo N. Bacterial and viral infections associated with influenza. Influenza Other Respir Viruses. 2013;7 Suppl 2:105-13. DOI: 10.1111/irv.12089.

62.Peng D, Zhao D, Liu J, Wang X, Yang K, Xicheng H, et al. Multipathogen infections in hospitalized children with acute respiratory infections. Virol J. 2009;6:155. DOI: 10.1186/1743-422X-6-155.

63.Sasaki A, Suzuki H, Saito R, Sato M, Sato I, Sano Y, et al. Prevalence of human metapneumovirus and influenza virus infections among Japanese children during two successive winters. Pediatr Infect Dis J. 2005;24(10):905-8.

64.Fischer WA, 2nd, Gong M, Bhagwanjee S, Sevransky J. Global burden of influenza as a cause of cardiopulmonary morbidity and mortality. Glob Heart. 2014;9(3):325-36. DOI:

10.1016/j.gheart.2014.08.004.

65.Beigel JH, Bao Y, Beeler J, Manosuthi W, Slandzicki A, Dar SM, et al. Oseltamivir, amantadine, and ribavirin combination antiviral therapy versus oseltamivir monotherapy for the treatment of influenza: a multicentre, double-blind, randomised phase 2 trial. Lancet Infect Dis. 2017;17(12):1255-65. DOI: 10.1016/S1473-3099(17)30476-0.

66. Hsu J, Santesso N, Mustafa R, Brozek J, Chen YL, Hopkins JP, et al. Antivirals for treatment of influenza: a systematic review and meta-analysis of observational studies. Ann Intern Med. 2012;156(7):512-24. DOI: 10.7326/0003-4819-156-7-201204030-00411.

67.Dobson J, Whitley RJ, Pocock S, Monto AS. Oseltamivir treatment for influenza in adults: a meta-analysis of randomised controlled trials. Lancet. 2015;385(9979):1729-37. DOI: 10.1016/S0140-6736(14)62449-1.

68.Centers for Disease Control and Prevention. Severe methicillin-resistant Staphylococcus aureus community-acquired pneumonia associated with influenza-Louisiana and Georgia, December 2006January 2007. MMWR Morb Mortal Wkly Rep 2007.

69.Derrar F, Voirin N, Khanafer N, Izri K, Gradi EA, Aitaissa A, et al. Influenza surveillance during the 2009-10, 2010-11, 2011-12 and 2012-13 seasons in algeria. J Med Virol. 2019. DOI: 10.1002/jmv.25469.

70.Hagerman A, Posfay-Barbe KM, Duppenthaler A, Heininger U, Berger C, Group PIS. Clinical characteristics and outcomes in children hospitalised with pandemic influenza A/H1N1/09 virus infection - a nationwide 
survey by the Pediatric Infectious Diseases Group of Switzerland. Swiss Med Wkly. 2015;145:w14171. DOI: 10.4414/smw.2015.14171.

71.Paul Glezen W, Schmier JK, Kuehn CM, Ryan KJ, Oxford J. The burden of influenza B: a structured literature review. Am J Public Health. 2013;103(3):e43-51. DOI: 10.2105/AJPH.2012.301137.

72.Hancock Shelley Zansky Gary E. Hollick Brian Fowler Christie McDonald-Hamm Ann Thomas Vickie Horan Mary Lou Lindegren William Schaffner Andrea Price Ananda Bandyopadhyay Alicia M. Fry SSSSCAPTDMPDKKY-HMMFMHRSRLCMEB. Comparing Clinical Characteristics Between Hospitalized Adults With Laboratory-Confirmed Influenza A and B Virus Infection Clinical Infectious Diseases. 2014;59(2):252-5.

73.Tran D, Vaudry W, Moore D, Bettinger JA, Halperin SA, Scheifele DW, et al. Hospitalization for Influenza A Versus B. Pediatrics. 2016;138(3). DOI: 10.1542/peds.2015-4643.

74.Allbrahim M, Assaf-Casals A, Massaad E, Shaker R, Soudani N, Fayad D, et al. Molecular epidemiology and genetic characterization of influenza B virus in Lebanon during 2016-2018. Infect Genet Evol. 2019;75:103969. DOI: 10.1016/j.meegid.2019.103969.

75.Azziz Baumgartner E, Dao CN, Nasreen S, Bhuiyan MU, Mah EMS, Al Mamun A, et al. Seasonality, timing, and climate drivers of influenza activity worldwide. J Infect Dis. 2012;206(6):838-46. DOI:

10.1093/infdis/jis467.

76.Caini S, El-Guerche Seblain C, Ciblak MA, Paget J. Epidemiology of seasonal influenza in the Middle East and North Africa regions, 2010-2016: Circulating influenza A and B viruses and spatial timing of epidemics. Influenza Other Respir Viruses. 2018;12(3):344-52. DOI: 10.1111/irv.12544.

\section{Figures}


Pre and post pandemic

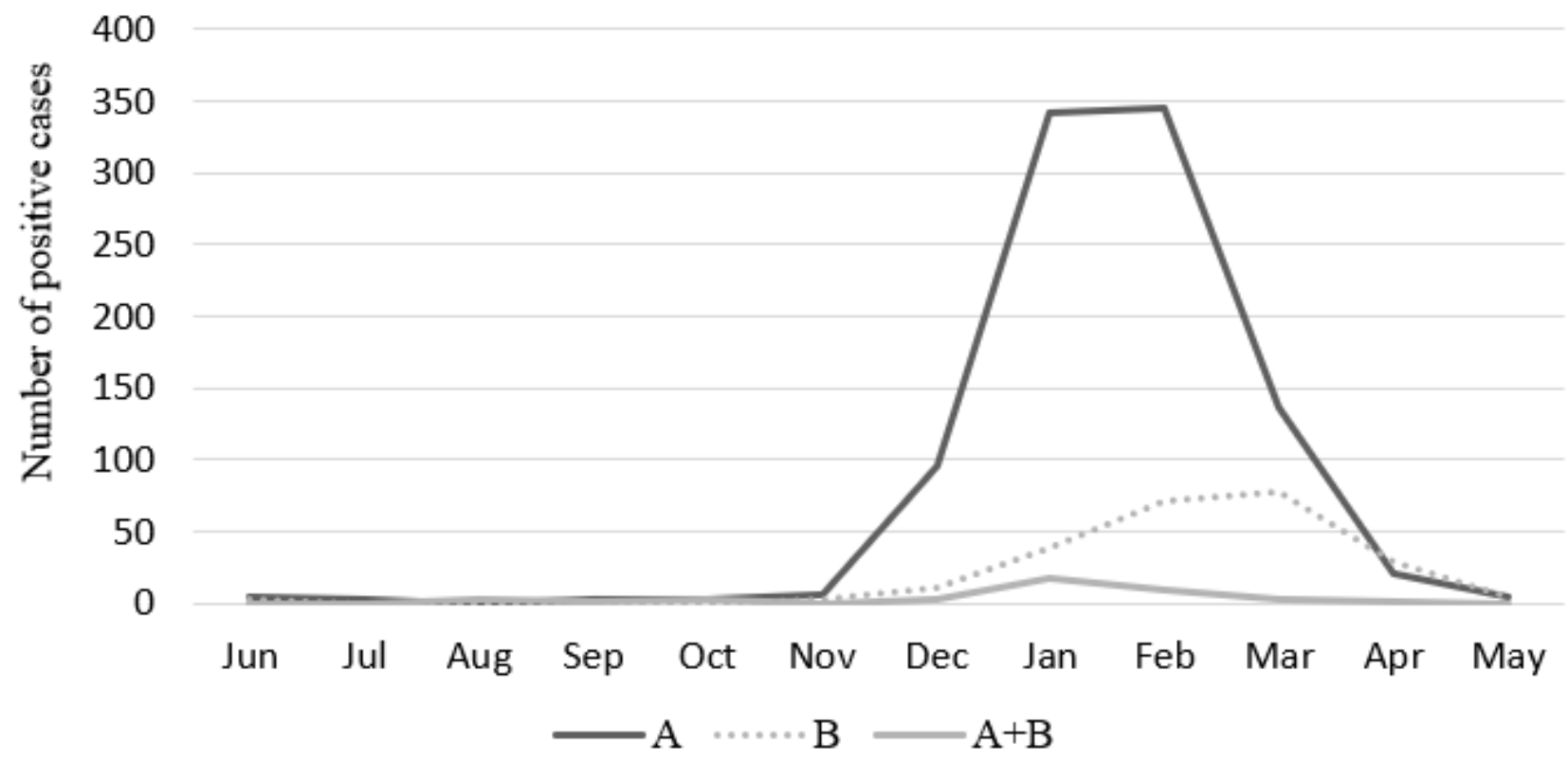

2009

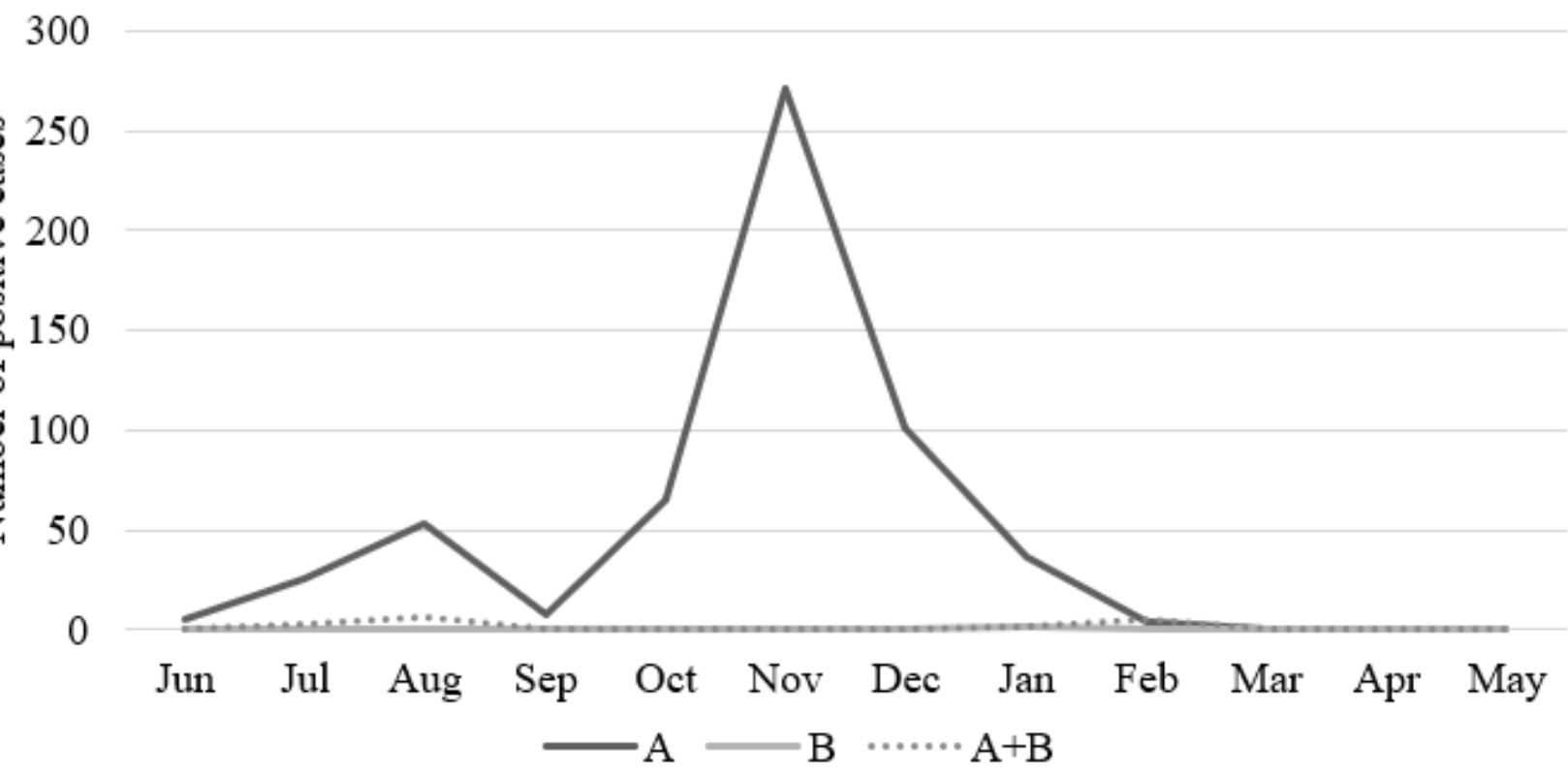

Figure 1

Influenza A and B virus circulation by month 


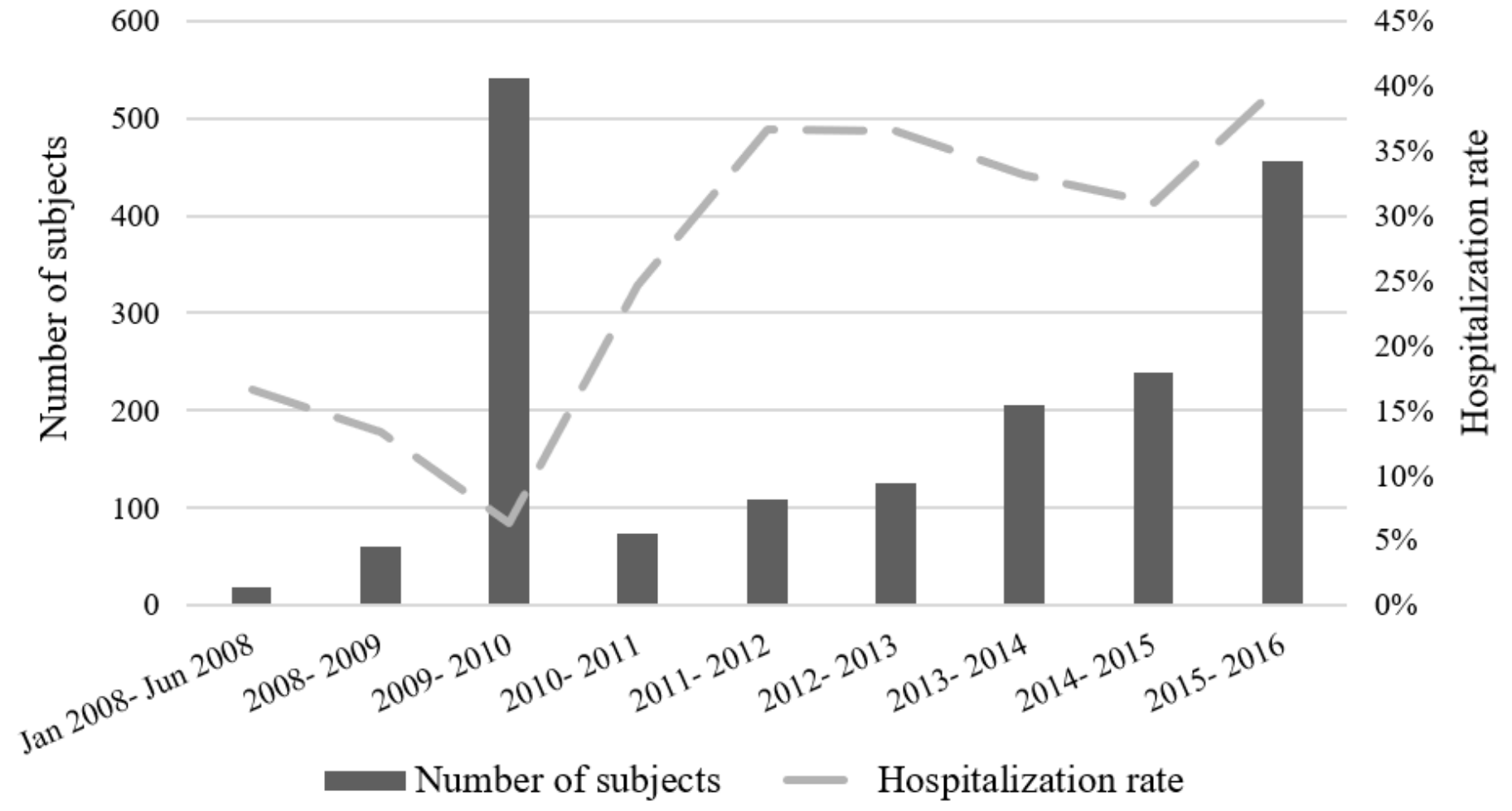

Figure 2

Influenza-associated hospitalization rate over the seasons

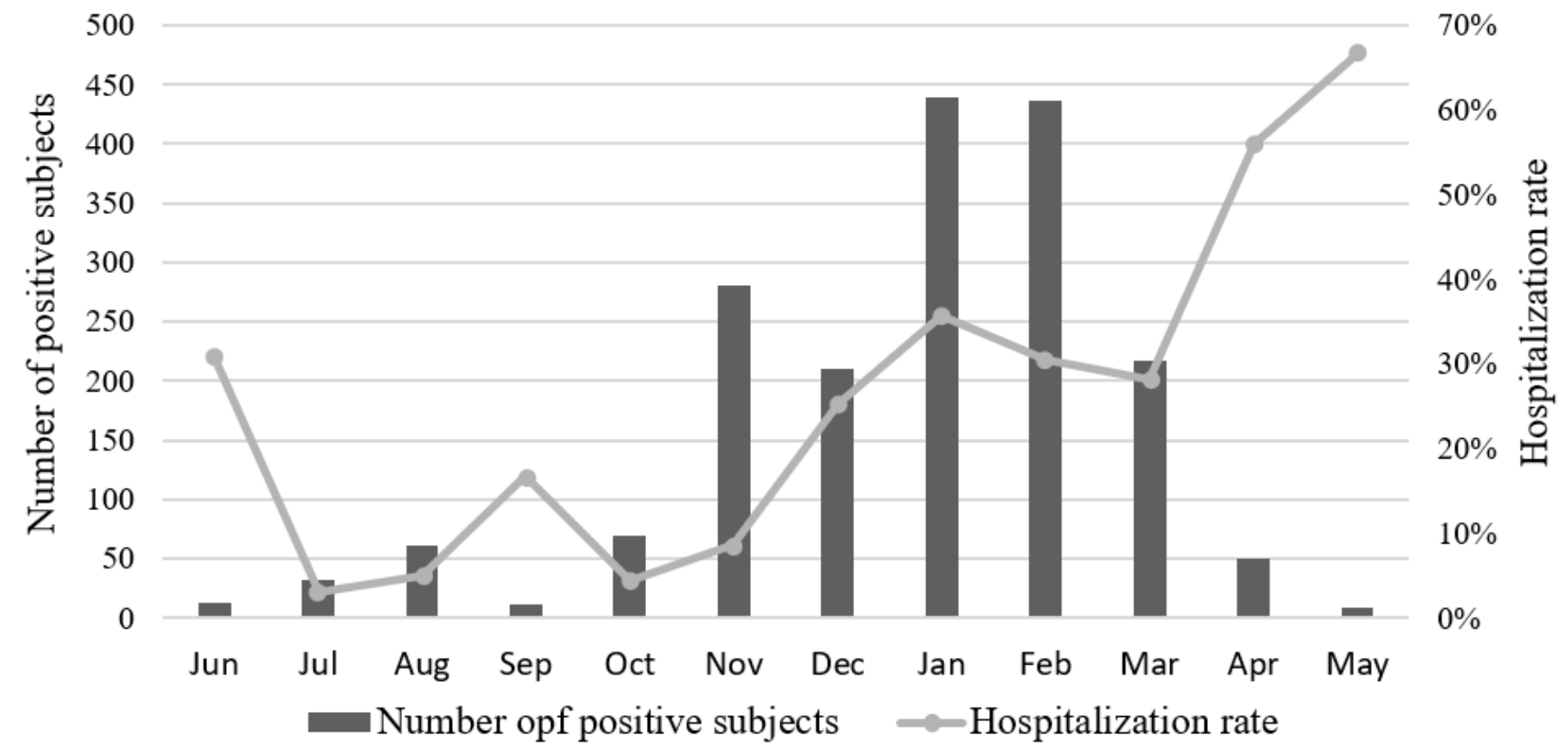

Figure 3

Influenza-associated hospitalization rate by month 